Miami Nature Biotechnology Short Reports

TheScientificWorld (2001) 1(S3), 78SR

ISSN 1532-2246; DOI 10.1100/TSW.2001.137

\title{
GENE EXPRESSION PROFILING OF HEALTHY CENTENARIANS
}

\author{
M. Venanzoni ${ }^{1,2}$, C. Franceschi ${ }^{1,3}$, S. Sorbi ${ }^{4}$, L. Storari ${ }^{1}$, S. Giunta ${ }^{1}$ and A. Seth ${ }^{5, *}$
}

${ }^{1}$ Laboratory of Molecular Oncology, INRCA, Ancona, Italy; ${ }^{2}$ ITBM, CNR, Rome, Italy; ${ }^{3}$ Univ. of

Bologna; ${ }^{4}$ Dept. of Neurology and Psychiatrics, Univ. of Firenze, Italy; ${ }^{5}$ Dept. of Laboratory Medicine and Pathobiology, and Laboratory of Molecular Pathology, Sunnybrook and Women's College Health Sciences Center, Univ. of Toronto, ON Canada

* Corresponding author.

INTRODUCTION. Human aging is characterized by a complex remodeling of immune, endocrine, metabolic parameters. In particular, organismal aging is characterized by high levels of proinflammatory molecules paralleling a progressive decay of response to stress at cellular and systemic level The few data on centenarian fibroblasts suggest that these cells possess only some features of replicative senescence, indicating that in vitro senescence plays a role, but do not fully mimic in vivo senescence. On the whole, aging is more appropriately to be considered as the consequence of the remodeling of an expression pattern involving a great number of genes. To compare the pattern of gene expression in centenarians vs. young control fibroblasts, an array of 384 genes was designed and cDNAs were robotically spotted. Genes were chosen on the basis of relationship with cell senescence, role in cellular stress response, involvement in apoptosis, genes involved in inflammation, tumor suppressor genes, cell cycle regulators, genes whose products have been demonstrated to change during aging, and genes whose genetic variants have been associated with human longevity.

METHOD. Primary cultures were established from skin fibroblasts from the forearms of 3 healthy female centenarians and 2 healthy female young controls (20 and 25 years old) from Central Italy. Total RNA was extracted by RNAzol B and further purified using Dynabeads coated with oligo-dT. Complex cDNA probes were produced by reverse transcription of mRNA followed by random primer labeling in presence of ${ }^{32} \mathrm{P}$-dCTP (1). Separate bacterial cultures harboring human cDNAs (I.M.A.G.E.) clones corresponding to known genes were robotically arrayed on nylon membranes (1). These were hybridized with the radiolabeled complex cDNA probes. The extent of hybridization at each spot was quantitated by phosphorimaging plate and by related software (1). Results are shown as intensity units for each cDNA relative to all other cDNAs.

RESULTS AND DISCUSSION. Max is one of the most up-regulated genes in fibroblasts from centenarians. Max forms heterodimers with Myc and regulates a variety of genes including telomerase, which affects the rate of aging in proliferative tissues. Stress response pathways were induced in centenarian fibroblasts as demonstrated by STAT, Heat Shock 70 and HSF expression. Surprisingly, p66SHC, the gene that affects life span in mice, is also expressed. DNA damage inducible genes, such as p53 and ATM, are induced, indicating that genotoxic stress occurs during aging. TGFb, TGFbR and activin receptors were induced, as was the IGF II receptor, an enhancer of TGF-beta response. Cell cycle related genes may be blocked, as $\mathrm{p} 57^{\mathrm{KIP} 2}$ was induced and Cyclins A, D1, F were down regulated, despite upregulation of cdk4 and p35 cyclin $\mathrm{H}$. Apoptosis may be promoted in centenarian fibroblasts 
given that Caspase3 was up-regulated, and the potent anti-apoptotic IL-15 was down-regulated (Table1).

Table 1. Greatest change in relative intensity units of Centenarian vs. Youth

\begin{tabular}{ll}
\hline Increased in Centenarian Fibroblasts & Decreased in Centenarian Fibroblasts \\
\hline p53 & Hic-5 \\
IGF-II receptor & Sm protein G \\
p35 cyclin H assembly factor & connective tissue GF \\
Max & PAK 1 \\
nerve growth factor receptor & BMP-1 \\
TRNR-2 & cyclin F \\
RNA helicase & TEK \\
Gos 3 [fosB] & abl interactor-2 (Abi-2) \\
alpha -1 type XVI collagen & LnRNP-C \\
(COL16A1) & \\
complement component 9 & cyclin A \\
complement component 8, beta & Mcl-1 \\
polypeptide & \\
Factor VII & type II serine/threonine kinase receptor \\
complement component 2 & IL-1 receptor type II \\
ATM & Sprk \\
ZO-1 & DLG-2 \\
Stat 5B & APO C III \\
Ski & jun D \\
heat shock factor 2 & IP gene \\
Id-1H & Thrombospondin \\
rap 1A & IL-10 receptor \\
\hline &
\end{tabular}

\section{REFERENCES.}

1. Seth, A., Lee, B.K., Qi, S., and Vary, C.P. (2000) J. Bone Miner. Res. 15(9), 1683-1696 

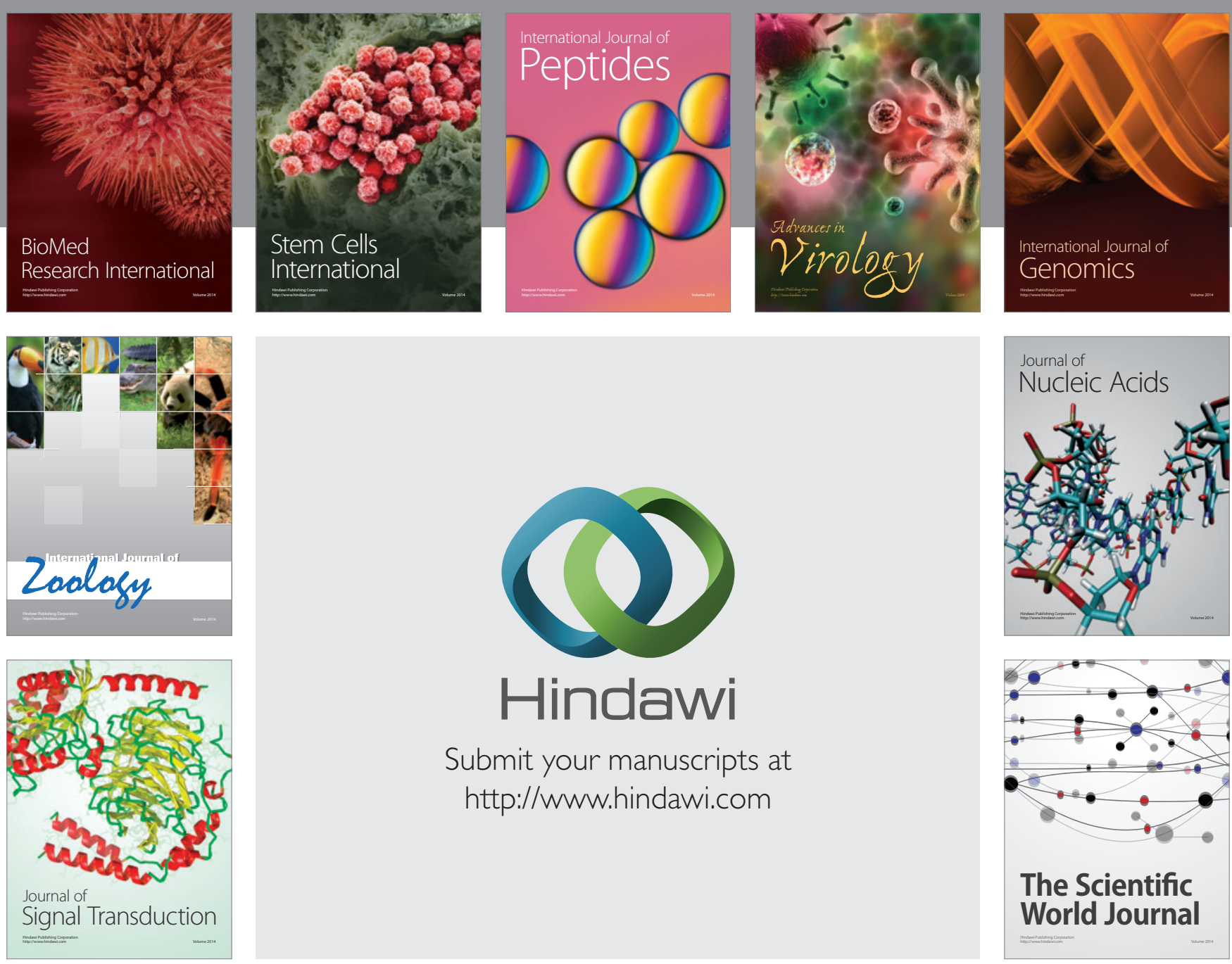

Submit your manuscripts at

http://www.hindawi.com
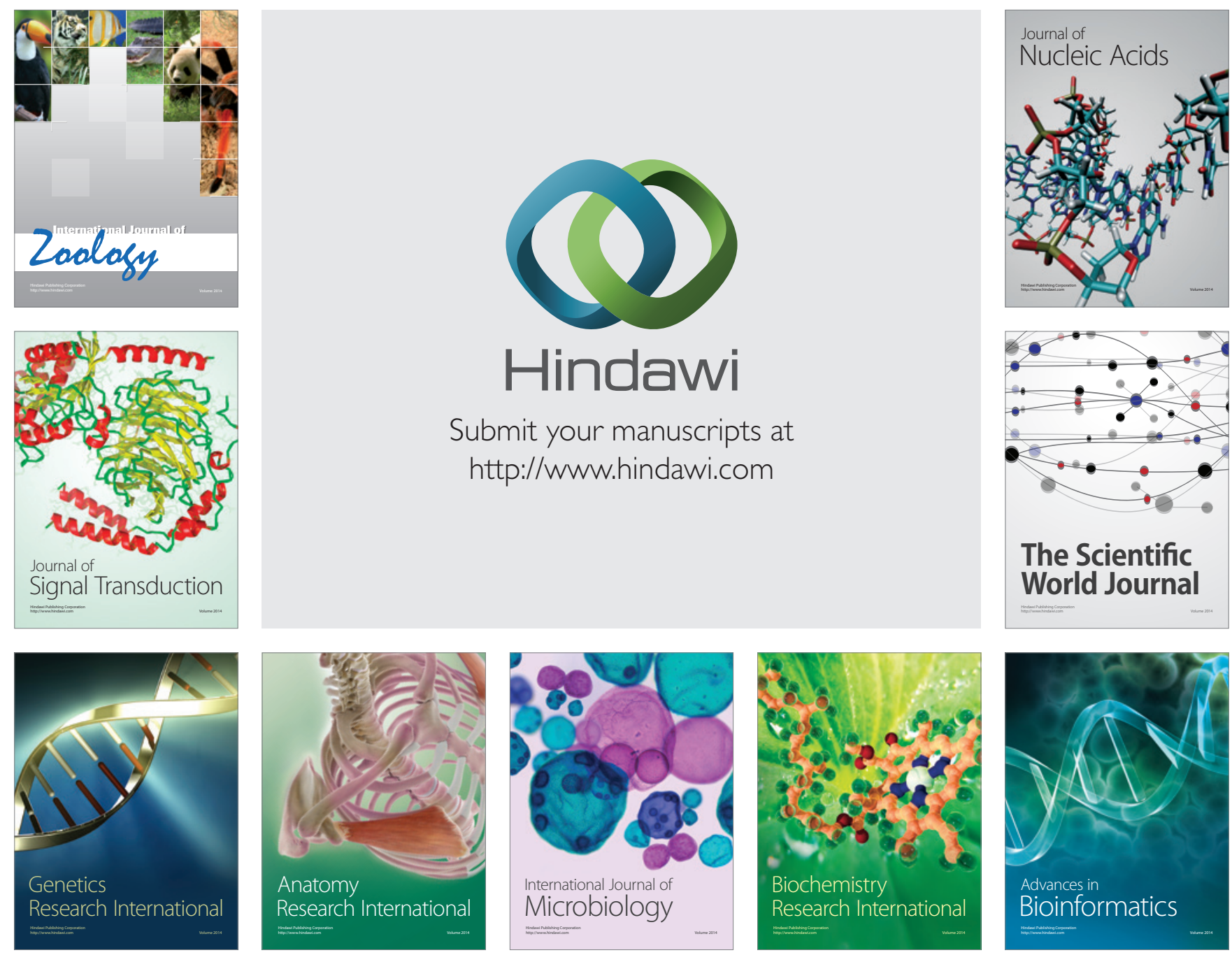

The Scientific World Journal
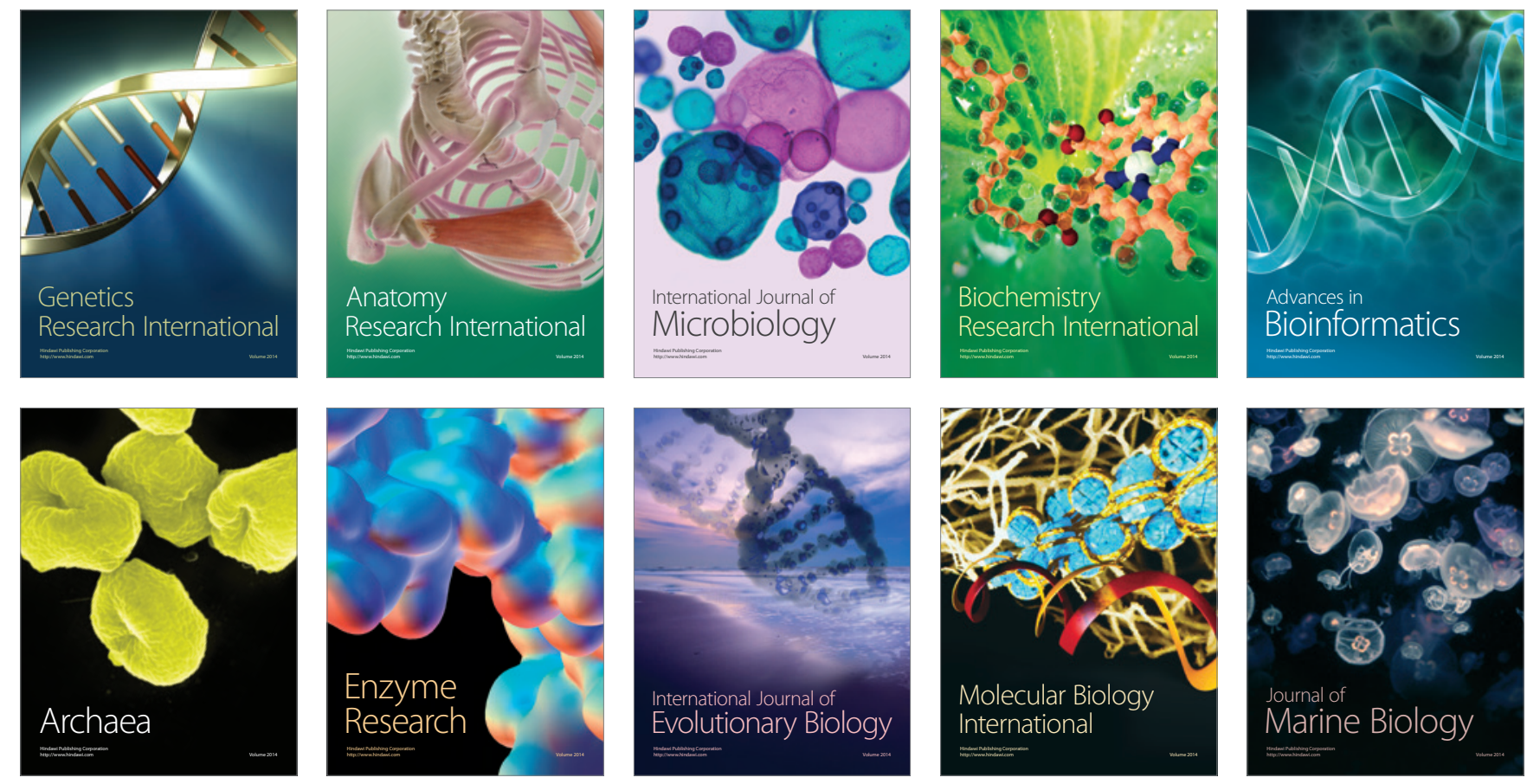